Raquel Aparecida Casarotto'

Luciane Frizo Mendes ${ }^{2}$

\title{
Queixas, Doenças Ocupacionais e Acidentes de Trabalho em Trabalhadores de Cozinhas Industriais
}

\author{
Complaint, Occupational Diseases \\ and Work Accidents Among \\ Industrial Kitchens Workers
}

Professora do Curso de Fisioterapia da Faculdade de Medicina da Universidade de São Paulo

'Professora do Curso de Fisioterapia da Universidade Metodista de São Paulo
As atividades desenvolvidas em cozinhas industriais apresentam um conjunto de características que podem desencadear doenças ocupacionais e acidentes de trabalho típicos. O objetivo deste estudo é comparar a prevalência de doenças ocupacionais, acidentes de trabalho e dores músculo-esqueléticas em trabalhadores de cinco cozinhas industriais. Para alcançar estes objetivos, foram avaliados 257 trabalhadores de cinco cozinhas industriais, com os seguintes procedimentos: entrevistas, questionários, análise da atividade real e aplicação do método NIOSH. Utilizou-se o teste qui-quadrado $(x)$ para comparar a homogeneidade dos dados entre os trabalhadores. Excetuando-se a prevalência de dor na região dorsal da coluna $(p=0,01)$ e alergia $(p=0,00461)$, todas as variáveis estudadas apresentaram $p>0,05$, mostrando a homogeneidade entre os trabalhadores. A análise ergonômica identificou os seguintes problemas: ambiente físico (dimensões, configurações, ambiências), instrumentos e equipamentos inadequados, má postura, organização do trabalho inadequada e levantamento de pesos acima dos limites recomendados.

Palavras-chaves Prevalência, Cozinha Industrial, Cozinheiros, Ergonomia, Análise do Trabalho.

The activities developed in industrial kitchens present a set of features that are able to trigger off typical occupational diseases and working accidents. The aim of this study is to compare the prevalence of occupational diseases, working accidents and musculoskeletal pain among workers of five industrial kitchens. In order to achieve these objectives, 257 workers were evaluated according to the following procedures: interviews, questionnaires, real activity analysis and the NIOSH method. We used the qui-square test to compare the prevalence of data among the workers. Except the pain in the dorsal spine $(p=0,01)$ and allergies $(p=0,0461)$, all the studied variables presented $p>0,05$, showing the homogeneity among the workers. The ergonomic analysis showed the following troubles: physical environment (dimensions, configurations, ambience), inadequate tools and equipment, bad posture and inappropriate work organization and excessive weight lifting.

Keywords Prevalence, Industrial Kitchen, Cooks, Ergonomics, Work Analysis. 


\section{Introdução}

As atividades desenvolvidas em cozinhas industriais caracterizam-se por manipulação manual intensa na preparação, durante o processo de servir os alimentos e limpeza do local de trabalho. A realização destas tarefas vem acompanhada de movimentos repetitivos de membros superiores e coluna, levantamento de pesos excessivos e permanência na postura em pé por períodos prolongados de tempo.

As doenças osteomusculares relacionadas ao trabalho (DORT) e os problemas de coluna podem desenvolver-se em atividades que apresentam estas características (Andersson, 1991; Keyserling, 1993; Sommerich, 1993; Ehrmann, 1996).

Estas patologias representam um dos principais problemas de saúde ocupacional que estão acometendo os trabalhadores no Brasil e no mundo nas três últimas décadas. Os dados do Ministério do Trabalho sobre a prevalência de doenças ocupacionais no Brasil, no ano de 2000, mostram que a DORT ocupa o primeiro lugar, seguida por dores na coluna, acompanhando a tendência mundial do aumento da incidência destas patologias. (English, 1995; Ribeiro, 1997; Settimi, 1998; Silverstein, 1997; Araújo, 1998.)

A porcentagem de trabalhadores ocupados no setor de alimentação na região metropolitana de São Paulo é de 1,3\% no setor industrial e 5,3 no setor de serviços (Seade, 2002).

Os estudos realizados com trabalhadores do setor alimentício mostram uma prevalência de Contact Dermatitis (Tacke, 1995), Trigger Finger (Gorsche, 1998), Carpal Tunnel Syndrome (Gorshe, 1999), Burns (Riina, 2000), Epicondylitis (Ono, 1998) nesta população, relacionando a doença à atividade ocupacional.

As doenças e acidentes de trabalho que podem acometer trabalhadores do setor alimentício, especificamente que realizam suas atividades em cozinhas industriais são inúmeros e pouco descritos, o que dificulta a realização de intervenções que diminuam a incidência e prevalência destes acometimentos e também o próprio atendimento médico e encaminhamento previdenciário correto dos trabalhadores adoecidos.
Assim, o objetivo deste estudo é analisar as condições de trabalho, comparar as queixas de dores e a prevalência de doenças e acidentes de trabalho identificadas em cinco cozinhas industriais.

\section{Material e métodos}

Este estudo realizou-se durante os anos de 1997 e 1999 nas cozinhas de quatro restaurantes universitários e de um hospital pediátrico em função do grande número de queixas e afastamentos de funcionários causados principalmente por DORT e Dores na Coluna. As nutricionistas responsáveis pela unidade solicitaram uma avaliação e sugestões para minimizar o impacto da atividade nos trabalhadores. Não haviam dados sistematizados de doenças e acidentes de trabalho registrados no setor que servissem de base para comparação com os dados obtidos nos questionários.

Sujeitos: Foram avaliados 186 trabalhadores das cozinhas de quatro restaurantes universitários e 71 da cozinha de um hospital pediátrico.

A função destes trabalhadores consistia na organização de cardápios e dietas, preparação de refeições, limpeza do ambiente de trabalho e dos utensílios utilizados, distribuição de refeições, armazenamento dos alimentos.

Procedimento: $O$ trabalho foi dividido em várias etapas. Inicialmente, realizaram-se reuniões com as nutricionistas para explicar o desenvolvimento do trabalho e para um entendimento da dinâmica do serviço. A partir de então, passou-se à análise das condições de trabalho, que constou dos seguintes procedimentos:

- Entrevistas individuais estruturadas com os funcionários mais novos, os mais antigos e com as nutricionistas responsáveis por cada unidade. Foram entrevistados 17 trabalhadores.

- Questionário sobre condições de trabalho, com questões abertas, fechadas e de múltipla escolha (Elias, 1982 e INRS, 1986). 199 trabalhadores responderam ao questionário, total ou parcialmente, que demorava cerca de 20 minutos para ser preenchido.

- Observação da situação real de trabalho no que diz respeito à organização, posturas, dimensionamento e ambiente dos pos- 
tos de trabalho, características dos utensílios e equipamentos. As observações aconteceram em todos os horários da jornada de trabalho, para avaliar as diferentes atividades desenvolvidas durante o dia de trabalho.

- Avaliação da Carga Limite Recomenda através do Método NIOSH (National Institute for Occupational Safety and Health) (Waters, 1993).

- Classificação de Repetitividade de acordo com os critérios do NIOSH (Putz-Anderson, 1991).

\section{Análise estatística}

Para comparar a prevalência de doenças ocupacionais, acidentes de trabalho e a localização das dores músculo-esqueléticas entre os trabalhadores das duas cozinhas estudadas, utilizou-se o teste qui-quadrado (X). Excetuando a dor na região dorsal $(p=0,01)$ e as alergias $(p=0,0461)$, em todas as outras variáveis estudadas verificou-se um valor de $p>0,05$, indicando que os grupos estudados apresentavam igualdade na prevalência de doenças, acidentes e desconfortos músculo-esqueléticos.

\section{Resultados}

A idade média dos funcionários era de 37,8 anos, com máxima de 64 e mínima de 21. O tempo médio de permanência no posto de trabalho era de 8,18 anos. As mulheres representavam $74 \%$ do total de trabalhadores. O gráfico 1 apresenta os acidentes de trabalho sofridos durante o desenvolvimento das atividades ocupacionais nas cozinhas estudadas.

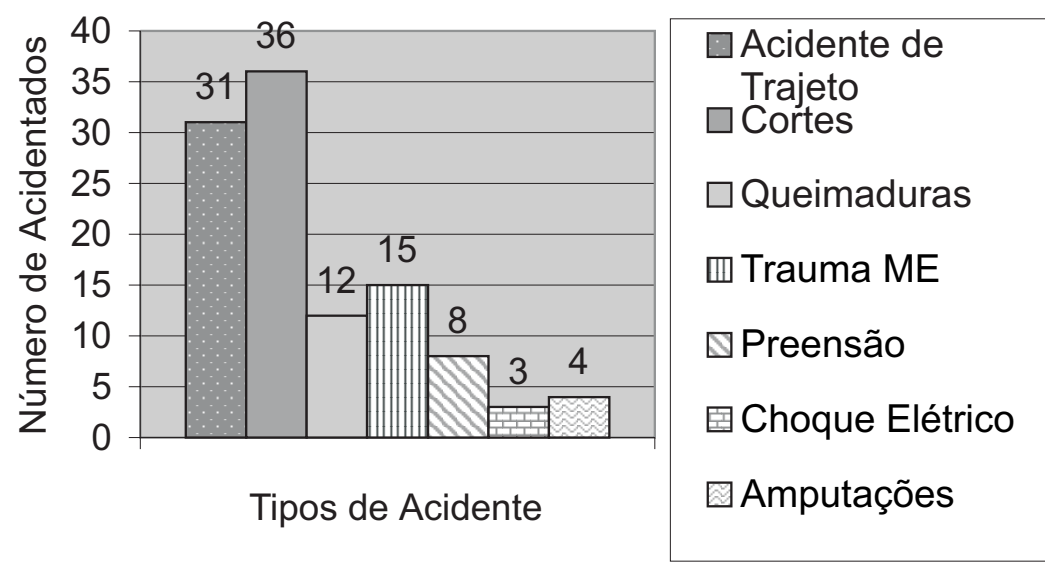

Gráfico 1 Número e tipos de acidentes de trabalho sofridos pelos trabalhadores das cozinhas industriais.

A tabela 1 apresenta as doenças ocupacionais mais citadas. Foram excluídas doenças não ocupacionais, embora fossem relatadas pelos trabalhadores, uma vez que - seu desenvolvimento ocorreu durante o período de trabalho nas cozinhas.

Tabela 1 Prevalência e tipo de doenças ocupacionais dos trabalhadores de cozinhas.

\begin{tabular}{c|c}
\hline $\begin{array}{c}\text { № de Trabalhadores } \\
\text { Acometidos }\end{array}$ & Tipos de Doenças ocupacionais \\
\hline 29 & DORT \\
28 & Dor na Coluna \\
6 & Distúrbios Psiquiátricos \\
4 & Doenças Reumáticas \\
4 & Alergias \\
4 & Distúrbios Visuais \\
3 & Distúrbios Auditivos \\
\hline
\end{tabular}


A tabela 2 apresenta o número de trabalhadores e as regiões com maior prevalência de queixas de desconforto postural.

Tabela 2 Prevalência de queixas de desconforto postural por região corporal.

\begin{tabular}{c|c}
\hline $\begin{array}{c}\text { No de } \\
\text { Trabalhadores } \\
\text { Acometidos }\end{array}$ & Regiões Corporais \\
\hline 111 & Dor nas Pernas \\
101 & Dor nos Pés \\
98 & Região Lombar \\
97 & Ombro D \\
76 & Ombro E \\
73 & Mão/Punho D \\
73 & Região Dorsal \\
71 & Braço D. \\
69 & Joelhos \\
69 & Nuca \\
53 & Braço E \\
53 & Mão/Punho E \\
37 & Coxas \\
20 & Nádegas \\
\hline
\end{tabular}

Os problemas relacionados ao ambiente de trabalho foram o ruído excessivo em 2 cozinhas de restaurantes universitários, devido ao grande número de caldeiras, exaustores barulhentos e válvulas de caldeiras quebradas. Com relação ao ambiente térmico, observouse umidade excessiva no verão, temperatura elevada próximo a fogões e auto-claves e baixas junto às câmaras frias. A ventilação é insatisfatória em todas as cozinhas avaliadas, devido a projetos inadequados no sistema de exaustão e circulação de ar.

O ambiente de trabalho apresentou alguns fatores que podem contribuir para acidentes de trabalho com o chão molhado e escorregadio, observados em 3 restaurantes universitários e na área de preparo de alimentos na cozinha do hospital. Um outro fator observado foi a possibilidade de queda de equipamentos sobre os trabalhadores, devido ao armazenamento inadequado de utensílios.

Com relação aos instrumentos nos postos de trabalho, verificou-se a presença de instrumentos pesados, com desenho antiergonômico e dimensionamento inadequado, que levava à adoção de posturas que proporcionam o aparecimento de LER/DORT (Putz-Andersson) e dores na coluna (Marras
2000), tais como:

- desvio ulnar e radial no uso de facas, espremedores de frutas, colheres e jarras.

- flexo-extensão de punho no manuseio de picadores, facas e colheres.

- prono-supinação com escumadeiras e conchas.

- Movimento de pinça dos dedos no envasamento de mamadeiras.

- Inclinação de tronco e força excessiva no manuseio de cubas e panelas.

- Força excessiva na utilização de facas não afiadas e escumadeiras com cabos longos, transporte de bandejas, manuseio do alicate de lacre das gavagens (peça onde são colocadas as mamadeiras para esterilização) e para empurrar os carrinhos prateleiras.

- Inclinação excessiva de tronco no manuseio de vassouras, rodos, pias e cubas.

- Elevação dos membros superiores acima de $90^{\circ}$ no manuseio de espremedores, no envasamento de mamadeiras, no preparo de alimentos no caldeirão a vapor, alcançar bandejas, retirar alimentos de estoques.

- Manuseio de equipamentos que provocam vibrações (espremedor de frutas, fatiador de legumes) 
- Permanência por períodos prolongados de tempo na postura em pé em todas as atividades, exceto na escolha de cereais, que é feita sentada.

- Os instrumentos de trabalho apresentavam alguns riscos no seu manuseio, como preensão de dedos na tampa das caldeiras (trava de tampas quebradas) e também amputações de dedos relacionadas à falta de utilização do êmbolo para empurrar a carne no moedor.

No item organização do trabalho, verificou-se que os ciclos de atividade foram classificados como repetitivos (ciclos de atividade menores que 30 segundos) nas atividades de servir e cortar alimentos, secar bandejas, escolher cereais, envasar mamadeiras ou com mais de $50 \%$ do tempo de trabalho utilizando os mesmos grupos musculares, nas atividades de secar louça, recepção de bandejas, descongelar alimentos, estocar alimentos em câmaras frias, preparar alimentos.

\section{A jornada de trabalho era de 8 h.}

Os funcionários faziam duas pausas para o café, com cerca de 10 minutos durante o período da manhã e da tarde e uma hora para intervalo do almoço.

Na cozinha dos restaurantes os funcionários denominados auxiliares de cozinha rodiziavam semanalmente entre 3 tarefas:

- o pré-preparo de alimentos e saladas

- distribuição de alimento, lavagem e secagem de bandejas

- abastecimento dos balcões de abastecimento de distribuição e lavagem de louças.

Na cozinha do hospital não há rodízio de tarefas.

O hospital pediátrico apresentava ainda riscos biológicos na área de lavagem de mamadeiras, principalmente relacionados à limpeza inadequada na pré-lavagem.

Os ambientes de trabalho apresentam semelhanças em relação à carga física e à organização do trabalho. O diferencial entre as duas unidades relaciona-se à carga mental de trabalho, pois na cozinha hospitalar os funcionários referem que existe um medo constante de erro na preparação das dietas, na quantidade e no tipo, que pode desencadear riscos graves para os pacientes. Os funcionários do lactário também ficam isolados dos demais, não possuindo contato com o meio externo durante a jornada, pois este ambiente necessita de esterilização.

Com relação a CLR, avaliada pelo método $\mathrm{NIOSH}$, os levantamentos de peso no hospital pediátrico revelaram que não havia situações de levantamento com carga acima do limite recomendado. Nas cozinhas dos restaurantes encontramos situações com pesos acima dos recomendados.

Tabela 3 Atividades realizadas pelos trabalhadores dos restaurantes com pesos e cargas limites recomendados.

\begin{tabular}{ccc}
\hline ATIVIDADES & Peso Levantado $(\mathrm{Kg})$ & CLR $(\mathrm{Kg})$ \\
\hline Descarregamento de Caixas Maracujá & 20 & 4,36 \\
Empilhamento de Sacos de Açúcar & 10 & 0 \\
Carregamento de Caixas de Mandioca & 25 & 8,63 \\
Descarregamento de sacos de Cebola & 25 & 19,38 \\
\hline
\end{tabular}

\section{Discussão}

As atividades desenvolvidas nas cozinhas industriais estudadas apresentam riscos ocupacionais de doenças e acidentes de trabalho semelhantes. A análise estatística evidenciou este fato, uma vez que apenas duas variáveis apresentaram um nível de significância menor que $0.05 \%$.

A falta de planejamento ergonômico no projeto das cozinhas avaliadas, nos utensílios de trabalho associadas à manutenção deficiente dos equipamentos utilizados é responsável pelo grande número de acidentes e doenças ocupacionais. 
Alguns acidentes de trabalho estão relacionados com o desenvolvimento da tarefa em si, como cortes e queimaduras. Outros relacionam-se à falta de manutenção adequada dos equipamentos, como choques elétricos, preensões e amputações. Outros ainda devem-se às condições ambientais inadequadas, como, excesso de água no chão, sistema de drenagem ineficiente e piso com material inadequado. Riina (2000), identificou que $8 \%$ dos pacientes atendidos em um centro de queimaduras eram trabalhadores de cozinhas, todas por escaldo em pés ou tornozelos.

Os fatores de riscos apontados como responsáveis pelo aparecimento de DORT estão presentes em todas as atividades desenvolvidas nas cozinhas, como ciclos de atividade menores que 30 segundos ou utilização dos mesmos grupos musculares durante mais de $50 \%$ do ciclo de trabalho, posturas inadequadas de membros superiores, força manual excessiva. Estes fatores estão presentes nas revisões realizadas por Ohlsson (1995), Keyserling (2000) e Malchaire (2000).

Em termos de desconforto postural, verificou-se que a postura em pé é responsável pela grande prevalência de dores nas pernas e pés. Talvez ela seja responsável pelas dores na coluna lombar, porém existem estudos que mostram que tanto em trabalhos desenvolvidos na postura em pé, quanto na postura sentada, pode ocorrer lombalgia. No estudo realizado por Krijnen (1997) para verificar os fatores de risco para insuficiência venosa crônica (IVC) em homens que trabalhavam na postura em pé, a queixa de dor nas pernas estava presente em $22 \%$ dos indivíduos sem IVC, enquanto a queixa de lombalgia era de $36 \%$.

Das doenças ocupacionais relatadas pelos trabalhadores, as DORT ocupam o primeiro lugar, seguidas por lombalgias. Estes dados concordam com as estatísticas do Ministério do Trabalho sobre doenças ocupacionais no Brasil, mostrando que as sinovites e tenossinovites e dorsalgia também ocupam o primeiro e o segundo lugar respectivamnete (Dataprev, 2000).

Entre as doenças ocupacionais estudadas em cozinheiros, a epicondilite tem uma prevalência de $11,5 \%$, enquanto os trabalhadores do grupo controle apresentavam $2,5 \%$ de prevalência (Ono, 1998). Halberg (1992) e Osório (1994) apontam grande prevalência de síndrome do túnel do carpo entre cozinheiros.

O uso de força e desvio ulnar / radial foram considerados significativos e preditores de prevalênçia de doenças musculoesqueléticas em trabalhadores de corte de carne (Malchaire, 1996). Estes fatores estão presentes na atividade de cozinheiros, como alimentos, cozinhar, moer, limpar.

Com relação às $C L R s$, observou-se que no empilhamento de sacos de açúcar, o que tornou a tarefa impossível de ser realizada foi o número de levantamentos por minuto. $O$ Método NIOSH aceita no máximo 13 levantamentos por minuto para atividades desenvolvidas em uma hora ou menos. Neste caso, 15 sacos eram levantados por minuto. Nos outros casos, a altura onde as caixas eram depositadas e os movimentos de rotação de tronco fizeram com que os valores caíssem.

Além dos aspectos relacionados ao mobiliário, instrumentos de trabalho, organização do trabalho, o questionário revelou que fatores relacionados à sobrecarga mental e stress estavam presentes nas atividades. medo de errar receitas e causar danos à saúde dos pacientes e a contaminação com louças sujas foram apontados como fatores de stress pelos funcionários do hospital. A dificuldade de relacionamento com a chefia e a falta de funcionários foram apontados por ambas as populações estudadas.

A utilização de questionários de auto-avaliação para obtenção de dados sobre acidentes, doenças e desconfortos deveu-se ao fato de existir um controle adequado de registros de doenças e acidentes de trabalho nas duas instituições estudadas. Silverstein et al (1997) comparando as diferentes fontes de dados para um sistema de vigilância epidemiológica em DORT, verificaram que $20 \%$ dos casos positivos identificados por examinadores universitários treinados não foram identificados pelos questionários. Por isso, o número de pessoas com algum grau de lesão talvez possa ser até maior do que o identificado pelos questionários aplicados no restaurante. Apesar disso, a autora considera que ele pode ser aplicado conjuntamente com uma lista de fator que predispõe os trabalhadores à DORT, no sentido de orientar intervenções ergonômicas para minimizar o impacto desta atividade sobre a saúde ocupacional dos trabalhadores. 
Os trabalhadores que desenvolvem suas atividades em cozinhas sofrem, como se pode verificar neste estudo, de falta de planejamento ergonômico nos postos, instrumentos e organização do trabalho, merecendo ações para minimizar impacto desta atividade sobre sua saúde.

No trabalho realizado por Cook (1999), as modificações ergonômicas realizadas na tarefa de embalagem de carnes diminuíram a sobrecarga de alguns grupos musculares dos membros superiores. Neste tipo de atividade ocupacional, todo o investimento é pouco, pois as condições de trabalho geram uma sobrecarga física importante nos trabalhadores. Parece que a tecnologia do século XXI ainda não chegou para estas atividades, causando uma enorme sobrecarga para estes trabalhadores.

\section{Referências bibliográficas}

Andersson, G.B. - The epidemiology of spinal disorders. In: Frymoyer, J.W. The Adult Spine: Principles and Practice. $1^{\mathrm{a}} \mathrm{ed}$. New York. Ed. Raven Press. 1991. p.107-146.

Amaral, F.G. O método NIOSH - Método prático para avaliar cargas e o risco dorso-lombar associado. Anais do Segundo Congresso Latino Americano e Sexto Congresso Brasileiro de Ergonomia, Florianópolis, Abergo, p.240-247, 1993.

Araújo, J.N.G. \& cols. L.E.R. Dimensões ergonômicas, psicológicas e sociais. São Paulo, Ed. Health, 1998.

Barreira, T.H.C., Abordagem ergonômica na prevenção da LER. Revista Brasileira de Saúde Ocupacional. São Paulo, 22 (84):51-60.1994.

Cook, T.M., Ludewig P.M., Rosecrance, J.H., Zimmermann, C.L., Gerleman, D.G. Electromyographic effects of ergonomic modifications in selected meatpeacking tasks. Applied Ergonomicas. 30:229233.1999.

Dataprev. Quantidade de acidentes de trabalho registrados, por motivo, segundo os 50 códigos da Classificação Internacional de doenças (CID) mais incidentes. 2000.

Ehrmann, F. D., Rossignol, M., Abehain, L, Gobeille,D. Physician referral to Physical Therapy in a cohort of workers compensated for Low Back Pain. Physical Therapy, 75(2):150-156, 1996.

English, C.J., Maclaren, W.M., Court-Brown, C., Hughes, S.P.F., Porter, R.W., Wallace, W.A., Graves, R.J., Pethick, A .J., Soutar, C.A. Relations between upper limb soft tissue disorders and repetitive movements at work. American Journal of Industrial Medicine. 27, p. 75-90, 1995.

Elias R. Conditions de travail devant les écrans cathodiques. Questionnaire d'evaluation. Cahiers de Notes Documentaires, nº 97. 1979.

Fundação Sistema Estadual de Análise de Dados (Seade). Documento eletrônico capturado em 23/07/2002.

Garcia, M.E.; Martins, A . B.K.; Hirata, E.E.; Braggio, E.F.; Rossi, J.C.B.; Albuquerque, V.G.C.; Rinaldo, I.; Rodrigues, L.E.A .; Nogueira, D.P.; Tolosa, E.M.C. Distúrbios ósteo-musculares relacionados ao trabalho na Coordenadoria de Assistência Social da Universidade de São Paulo. Revista do Hospital Universitário, 8(1), p.2124. 1998.

Gorsche, R., Wiley, J. P., Renger, R., Brant, R., Gemer, T. Y. e Sasyuniuk, T.M. Prevalence and Incidence of Stenosing Flexor Tenosynovitis (Trigger Finger) in a Meat-Packing Plant. Journal Occupational and Environmental Medicine, 40(6), p.556-560. 1998. 
Gorsche, R., Wiley, J. P., Renger, R., Brant R., Gemer, T. Y. e Sasyuniuk, T.M. Prevalence and incidence of carpal tunnel syndrome in a meat packing plant. Occup Environ Med 1999; 56: 417-422.

Halberg, M., Morgenstem, H. Kelsh, M. Impact of occupational and job tasks on the prevalence of carpal tunnel syndrome. Scandinavian Journal of Work and Environment Health. 18: 337-45.1992.

Institut National de Recherche et de Sécurité (INRS). Les ècrans de Visualization, p.60,1986.

Keyserling, W.M., Stetson, D.S., Silverstein, B.A ., Brouwer, M.L. A checklist for evaluating ergonomic risk factors associated with upper extremity cumulative trauma disorders. Ergonomics, 36(7), p.807-831, 1993.

Keyserling W.M. Workplace risk factors and occupational musculoskeletal disorders, part 2: a review of biomechanical and psychophysical research on risk factors associated with upper extremity disorders. AlHAJ, 61:231-243.2000.

Krïnen, R.M.A, de Bôer E.M., Adèr H.J. Bruynzeel D.P. Venous Insufficiency in male workers with a standing profession. Dermatology, 194:111-120. 1997.

Maciel, R.H. Ergonomia e lesões por esforços repetitivos. In: Codo, W e Almeida, M.C.C.G. LER. Lesões por esforços repetitivos. Petrópolis, Ed.Vozes. p.163201. 1995.

Malchaire, J.B., Cook, N.A, Robert, A R. Prevalence of musculoskeletal disorders at the wrist as a function of angles, forces, repetitiveness and movement velocities. Scandinavian journal of work and Environment Health. 22:176-81.1996.

Malchaire, J. , Cock N., Vergracht, S. Review of the factors associated with musculoskeletal problems in epidemiological studies. International Archivies of Occupational and Environment Health. 74: 79-90.2001.
Marras, W.S. Occupational low back pain disorder causation and control. Ergonomics.34(7):880-902.2000.

Medeiros, E.A .M., Burbarelli, N.T.C. e Garbin. A . L. Prevenção de LER: Estudo de caso no telemarketing ativo da Abril S/A. Anais do $4^{\circ}$ Congresso Latino Americano de Ergonomia e $8^{\circ}$ Congresso Brasileiro de Ergonomia. Florianópolis, Abergo, p.312-315. 1997.

Ohlsson, K., Attewell, R.G., Palsson, B., Karlsson, B., Balogh, I., Johnsson, B., Ahlm, A ., Skerfving, S. Repetitive industrial work and neck and upper limb disorders in females. American Journal of Industrial Medicine. 27:731-747.1995.

Ono, Y., Nakamura, R., Shimaoka, M., Hiruta, S., Hattori, Y., Ichihara, G., Kamijima, M., Takeuchi, Y. Epicondylitis among cooks in nursery schools. Occup Environ Med 1998; 55: 172-179.

Osorio, A M., Ames, R.G., Jones, J., Castorina J., Rempel, D., Estrin W., Thompson, D. Carpal tunnel syndrome among grocery store workers.American journal of industrial medicine. 25: 229245. 1994.

Riina, L.H, Simpson, R.L., Gudjonsson, O., Glickman, L.T., Harris, S.U., Johnson, D., Ginocchio, M. Burn injury in kitchen workers: a cause for prevention. Journal of Burn Care \& Rehabilitation. nov/ dez.563-564. 2000.

Settimi, M.M. \& cols. Lesões por esforços repetitivos/ distúrbios ósteo-musculares relacionados ao trabalho: abordagem interdisciplinar. Rede Especial - revista do projeto de Cooperação Técnica Brasil-Itália. "Proteção a saúde nos ambientes de trabalho", p.149-153, 1998.

Silverstein, B.A . , Stetson, D.S., Keyserling, W.M., Fine, L.J. Work-related musculoskeletal disorders: comparison of data sources for surveillance. American Journal of Industrial medicine, 31, p. 600-608. 1997. 